\title{
Evaluation du pouvoir disjonctif des variables catégorielles impliquées dans la sélection du palmier à huile (Elaeis guineensis jacq.) en Côte d'Ivoire
}

\author{
Désiré ALLOU1, $2^{*}$, Benjamin ADON², Eugene KONAN2, Tristan DURAND- \\ GASSELIN ${ }^{3}$, Simon-Pierre N'GUETTA Assanvo' et Abdourahamane SANGARE ${ }^{2}$ \\ 'Laboratoire de génétique, Université de Cocody, UFR des Biosciences \\ 22 B P 723 Abidjan 22, Tel (225), Côte d'Ivoire \\ ${ }^{2}$ Centre National de Recherche Agronomique, 01 B P I740 Abidjan 01, Tel(225) 2347 \\ 2424 Fax (225) 234724 II, Côte d'Ivoire \\ ${ }^{3}$ CIRAD, TA 80/02, A venue Agropolis F34398 Montpellier CEDEX 5 - FRANCE
}

*Correspondance,courriel : desire_allou@yahoo.fr

\section{Résumé}

En amélioration génétique du palmier à huile (Elaeis guineensis Jacq.), les variables catégorielles constituent une part majoritaire de l'information utilisée pour différencier, les populations de géniteurs issus du programme de sélection récurrente réciproque (SRR). Le pouvoir disjonctif de ces variables a été testé sur 134 géniteurs sélectionnés issus des populations du premier, deuxième et troisième cycle de SRR. Les variables catégorielles utilisées pour différencier ces populations sont celles qui ont permis d'avoir des informations sur les origines géographiques et les généalogies parentales des géniteurs. L'analyse factorielle de l'ensemble de ces variables catégorielles, a permis de déterminer le pouvoir disjonctif de chacun des deux principaux critères de divergences des populations de géniteurs sélectionnes. Le critère "origine géographique" a représenté $14 \%$ d'expression de disjonction totale des populations de géniteurs sélectionnés. Le critère "généalogie parentale" a représenté $70 \%$ d'expression de disjonction totale des populations de géniteurs sélectionnés. L'utilisation de ces deux critères de différenciation des populations de géniteurs sélectionnées peut constituer une bonne alternative à l'utilisation des données moléculaires dans les études de la diversité des populations de palmier à huile en sélection.

Mots-clés : Palmier à huile, sélection, variable catégorielle, disjonction de population 


\section{Abstract \\ Assessment of the disjunctive power of categorical variable implied in the selection of oil palm (Elaeis guineensis jacq.) in Côte d'Ivoire}

In genetic improvement of oil palm (Elaeis guineensis Jacq.), categorical variable represented a main part of information used to determine differentiation among populations of genitors selected from the reciprocal recurrent selection program (RRS). The disjunctive power of these variables has been tested on 134 genitors selected from populations of the first, second and third cycle of RRS. The categorical variables used to differentiate these populations are those that permitted to have some information on the geographical origins and the parental genealogies of those genitors. The factorial analysis of those categorical variables, permitted to assess to the disjunctive power of each of the two main criteria's of divergences of select genitors populations. The criteria "geographical origin" represented $14 \%$ of total disjunction expression of selected parents' populations. The criteria "parental genealogy" represented $70 \%$ of total disjunction expression of selected parents' populations. The use of these two criteria's can constitute one fine alternative to the use of molecular data in diversity studies of oil palm selected populations.

Keywords : Oil palm, selection, categorical variable, disjunction of population,

\section{Introduction}

Le palmier à huile (Elaeis guineensis Jacq) est une monocotylédone de la famille des palmacées. C'est la plante oléagineuse la plus productive d'huile à l'hectare. En termes de volume mondial de production de corps gras d'origine végétale, l'huile de palme occupe la seconde place après l'huile de soja [1]. Cette performance est le résultat d'un programme de sélection de géniteurs basé sur un schéma de sélection récurrente réciproque [2]. Dans ce programme de sélection, des populations de géniteurs d'origine géographiques diverses ont été identifiées par différents centres de recherche. La diversité de ces populations de géniteurs a été évaluée par des critères agromorphologiques [3-5] et moléculaires [6-8]. Dans le cas des populations de géniteurs d'origines géographiques semblables et de performances agronomiques identiques, les disjonctions entre ces populations de géniteurs sélectionnés sur la base exclusive des critères agro-morphologiques ne peuvent plus être établies que sur des critères généalogiques. Les sélectionneurs du palmier à huile ont alors introduit l'usage de données catégorielles [9] de source généalogique $[10 ; 11]$. Ces données catégorielles constituées sous forme de données alphabétique, ont constitué une part importante 
dans l'information utilisée pour différencier les populations des géniteurs sélectionnés à l'aide d'autres critères. Cependant, en l'absence d'évaluation statistique du pouvoir disjonctif de ces variables catégorielles, l'exploitation de l'information sur le niveau de différenciation exprimé a été laissée aux seules appréciations des sélectionneurs. L'objectif de cette étude est d'évaluer, grâce à une analyse factorielle, le pouvoir de disjonction de différentes variables catégorielles couramment utilisées en amélioration génétique du palmier à huile. L'analyse en composante principale a été alors appliquée à un ensemble de variables catégorielles suggestives de différences entre populations de géniteurs sélectionnés issues des trois premiers cycles de sélection du palmier à huile en Côte d'Ivoire.

\section{Matériel et méthodes}

\section{2-1. Matériel végétal}

Tableau 1: Liste des géniteurs échantillonnés pour l'analyse de la divergence catégorielle.

\begin{tabular}{|c|c|c|c|}
\hline Cycle & $\begin{array}{l}\text { Croisement } \\
\text { réalisé }\end{array}$ & $\begin{array}{c}\text { Population } \\
\text { sélectionnées }\end{array}$ & $\begin{array}{l}\text { Nombre } \\
\text { géniteurs }\end{array}$ \\
\hline \multirow{2}{*}{1} & DABOU & $\mathrm{DA}$ & 07 \\
\hline & SOCFIN & SO & 03 \\
\hline \multirow{4}{*}{2} & DABOU x DABOU & DADA & 26 \\
\hline & DABOU X SOCFIN & DASO & 08 \\
\hline & SOCFIN x DABOU & SODA & 09 \\
\hline & ANGOLA x ANGOLA* & ANAN & 07 \\
\hline \multirow{8}{*}{3} & (DABOU x DABOU) $\times($ DABOU $\times$ DABOU) & DADADADA & 19 \\
\hline & (DABOU x DABOU) $\times($ DABOU $\times$ SOCFIN) & DADADASO & 02 \\
\hline & (DABOU x SOCFIN) $\times($ DABOU $\times$ DABOU) & DASODASO & 06 \\
\hline & (DABOU x SOCFIN) $\times$ (SOCFIN x DABOU) & DASOSODA & 05 \\
\hline & (SOCFIN x DABOU) x (DABOU x SOCFIN) & SODADASO & 03 \\
\hline & (SOCFIN x DABOU) x (SOCFIN x DABOU) & SODASODA & 06 \\
\hline & (DABOU $\times$ DABOU) $\times($ ANGOLA $\times$ ANGOLA) & DADAANAN & 17 \\
\hline & (DABOU x SOCFIN) $\times($ ANGOLA $\times$ ANGOLA) & DASOANAN & 02 \\
\hline
\end{tabular}

* les géniteurs d'origine Angola ont été introduits dans le programme d'amélioration génétique au 2 cycle de SRR

Le matériel végétal utilisé pour évaluer le pouvoir disjonctif des variables catégorielles a été constitué de 134 géniteurs caractérisés par une production de petit nombre de 
gros régimes regroupés en 14 populations. Ces populations de géniteurs sont issues de croisements entre palmier à huile de diverses origines géographiques (ANGOLA et DABOU originaire d'Afrique et SOCFIN originaire d'Asie). Les croisements ont été réalisés dans le premier cycle de sélection (2 populations), dans le deuxième cycle de sélection (4 populations) et dans le troisième cycle de sélection (8 populations) (Tableav 1).

\section{2-2. Méthodes}

\section{2-2-1. Variables catégorielles}

Deux critères fréquemment utilisés en sélection du palmier à huile ont servi à l'élaboration des variables catégorielles. Le critère "origine géographique" a été utilisé pour établir des différences entre populations à partir de la provenance des parents (Tableau 2). Le critère "généalogie parentale" a été utilisé pour établir des différences entre populations à partir des croisements des parents (Figure $\Pi$ ).

Tableau 2 : Variables catégorielles correspondant aux descripteurs de différences entre populations de géniteurs de palmier à huile.

\begin{tabular}{|c|c|c|c|}
\hline Critère & descripteur utilisé pour la disjonction & $\begin{array}{l}\text { Codification } \\
\text { variable }\end{array}$ & $\begin{array}{l}\text { Nombre } \\
\text { catégories }\end{array}$ \\
\hline $\begin{array}{l}\text { Origine } \\
\text { géographique }\end{array}$ & $\begin{array}{l}\text { Origine géographique du } 1^{\text {er }} \text { grand parent femelle } \\
\text { Origine géographique du } 1^{\text {er }} \text { grand parent mâle } \\
\text { Origine géographique du } 2^{\text {ème }} \text { grand parent femelle } \\
\text { Origine géographique du } 2^{\text {ème }} \text { grand parent mâle }\end{array}$ & $\begin{array}{l}\text { ORGDPAIF } \\
\text { ORGDPAIM } \\
\text { ORGDPA2F } \\
\text { ORGDPA2M }\end{array}$ & $\begin{array}{l}03 \\
03 \\
03 \\
03\end{array}$ \\
\hline $\begin{array}{l}\text { Généalogie } \\
\text { parentale }\end{array}$ & $\begin{array}{l}\text { Descendance des } 1^{\text {ers }} \text { grands-parents } \\
\text { Descendance des } 2^{\text {èmes }} \text { grands-parents } \\
\text { Génotype } 1^{\text {er }} \text { grand parent femelle } \\
\text { Génotype parental du } 1^{\text {er }} \text { grand parent mâle } \\
\text { Génotype parental du } 2^{\text {e }} \text { grand parent femelle } \\
\text { Génotype parental du } 2^{\text {ème }} \text { grand parent mâle } \\
\text { Descendance parentale } \\
\text { Parent femelle } \\
\text { Parent mâle } \\
\text { Combinaison parental }\end{array}$ & $\begin{array}{l}\text { DESGDPAI } \\
\text { DESGDPA2 } \\
\text { GRDPARIF } \\
\text { GRDPAR1M } \\
\text { GRDPAR2F } \\
\text { GRDPAR2M } \\
\text { DESCPAR } \\
\text { PARENTI } \\
\text { PARENT2 } \\
\text { TYPE }\end{array}$ & $\begin{array}{l}17 \\
15 \\
14 \\
11 \\
12 \\
09 \\
55 \\
40 \\
34 \\
14\end{array}$ \\
\hline
\end{tabular}

Pour chaque variable catégorielle élaborée, l'information recueillie sur un géniteur est une donnée alphabétique qui spécifie son appartenance à une catégorie de 
géniteurs sélectionnés. Chaque donnée a été ensuite codifiée de 100 à $100+(n-1)$, n étant le nombre total de catégories ou classes révélées, pour l'ensemble des géniteurs, par la variable catégorielle utilisée.

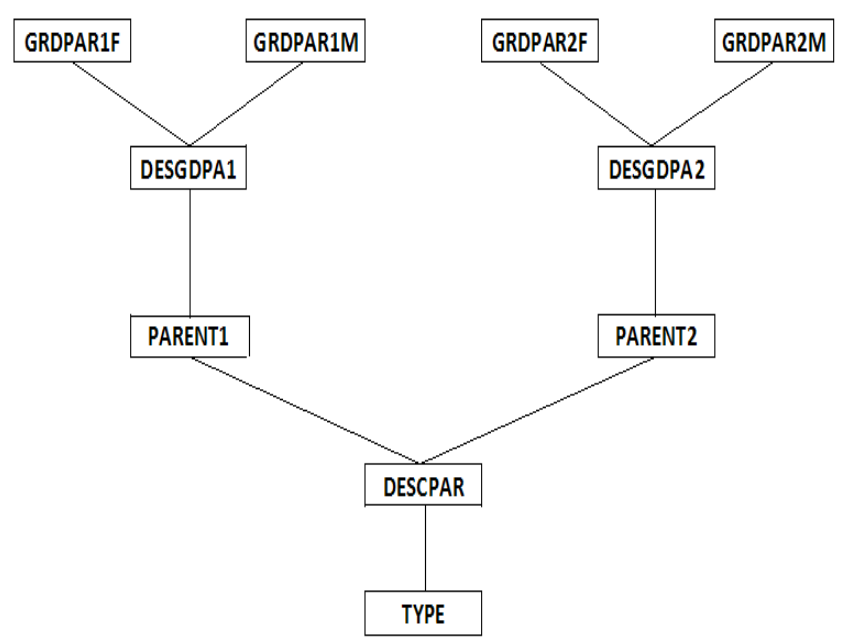

Figure 1 : Schématisation de la généalogie parentale d'un type de combinaison parental (géniteur) du $3^{\circ}$ cycle de SRR avec les variables catégorielles utilisées en sélection du palmier à huile.

\section{2-2-2. Analyse statistiques}

L'Analyse en Composantes Principales (ACP) a été effectuée pour identifier les valeurs des deux critères de disjonction des populations de géniteurs sélectionnés [12] ainsi que celle de leurs composantes [13]. La Classification Hiérarchique Ascendante (СAH) a été ensuite utilisée pour évaluer le pouvoir disjonctif des composantes du critère "généalogie parentale" en identifiant celles qui favorisent les meilleures disjonctions des huit populations de géniteurs du $3^{\text {ème }}$ cycle de SRR [14]. Ces analyses ont été effectuées avec la version 5.1 du logiciel STATISTICA [15]. 


\section{Résultats}

\section{3-1. Valeurs des critères de disjonction}

L'Analyse en Composante Principale a révélé que le critère "généalogie parentale" permet de déterminer cinq fois plus de disjonction que le critère "origine géographique". Sur l'axe 1 de l'ACP, le critère "généalogie parentale" a révélé $70 \%$ de la variabilité totale des populations de géniteurs sélectionnés. Les composantes les plus expressives de ce critère sont le "type parental", les "descendances des grands-parents", les "grands-parents", la "descendance parentale" et les "parents" (Tableau 3).

Tableau 3: Poids factorie/ des variables analysés en composantes principales dans la population de géniteurs sélectionnés du programme de SRR du palmier à huile.

\begin{tabular}{llll}
\hline \hline & AXE 1 & AXE 2 & AXE 3 \\
\hline TYPE & $\mathbf{0 , 9 7}$ & 0,09 & $-0,01$ \\
ORGDPAIF & 0,22 & $-\mathbf{0 , 8 8}$ & 0,41 \\
ORGDPA1M & 0,22 & $-\mathbf{0 , 8 8}$ & 0,41 \\
ORGDPA2F & 0,41 & $-0,40$ & $-\mathbf{0 , 8 1}$ \\
ORGDPA2M & 0,42 & $-0,46$ & $-\mathbf{0 , 7 8}$ \\
DESGDPA1 & $\mathbf{0 , 9 8}$ & 0,09 & $-0,06$ \\
DESGDPA2 & $\mathbf{0 , 9 4}$ & 0,11 & 0,22 \\
GRDPARIF & $\mathbf{0 , 9 6}$ & 0,09 & 0,16 \\
GRDPAR1M & $\mathbf{0 , 9 7}$ & 0,01 & 0,06 \\
GRDPAR2F & $\mathbf{0 , 9 6}$ & 0,09 & 0,19 \\
GRDPAR2M & $\mathbf{0 , 9 6}$ & $-0,01$ & 0,09 \\
DESCPAR & $\mathbf{0 , 9 7}$ & 0,09 & $-0,04$ \\
PARENT1 & $\mathbf{0 , 9 7}$ & 0,11 & $-0,09$ \\
PARENT2 & $\mathbf{0 , 9 8}$ & 0,10 & $-0,01$ \\
\hline Variance exprimée & $\mathbf{9 , 7 6}$ & 2,00 & 1,74 \\
Proportion Totale & 0,70 & 0,14 & 0,12 \\
\hline \hline
\end{tabular}

Sur l'Axe 2 de l'ACP le critère "origine géographique" a révélé $14 \%$ de la variabilité totale des populations de géniteurs sélectionnés. Les composantes les plus expressives de ce critère sont les "origines des premiers grands-parents". L'Axe 3 de l'ACP a donné $12 \%$ de la variabilité totale des populations de géniteurs sélectionnés. Les composantes les plus expressives de ce critère sont les "origines 
des deuxièmes grands-parents". Ces composantes ont exploité également la divergence basée sur le critère "origine géographique".

\section{3-2. Pouvoir disjonctif du critère "origine géographique"}

Les critères "origine géographique" a permis d'établir dans chacun des niveaux de sélection, des disjonctions dans les populations de géniteurs sélectionnés (Figure 2). Les populations de géniteurs du premier cycle de SRR ont été principalement séparées en deux catégories disjonctives en fonction du critère "origine géographique". La première catégorie disjonctive est composée de géniteurs dont les grands-parents sont d'origine DABOU. La deuxième catégorie disjonctive est composée de géniteurs dont les grands-parents sont d'origine SOCFIN. Les populations de géniteurs du deuxième cycle de SRR ont été séparées en trois catégories disjonctives. La première catégorie disjonctive est composée de géniteurs mono-origine dont les grands-parents sont d'origine ANGOLA. La deuxième catégorie disjonctive est composée de géniteurs mono-origine dont l'origine géographique des grands-parents est DABOU.

La troisième catégorie est formée des géniteurs bi-origine dont les grands-parents sont d'origines DABOU et SOCFIN. Les populations de géniteurs du troisième cycle de SRR ont été séparées en quatre catégories disjonctives. La première catégorie est constituée des géniteurs mono-origine dont les grands-parents sont tous d'origine DADOU. La deuxième catégorie disjonctive est composée de géniteurs bi-origines dont les grands-parents sont d'origines DABOU et SOCFIN. La troisième catégorie est composée des géniteurs bi-origines dont les grands-parents sont d'origines DABOU et ANGOLA. La quatrième catégorie est composée de géniteurs tri-origines dont les grands-parents sont d'origine DABOU, SOCFIN et ANGOLA. 


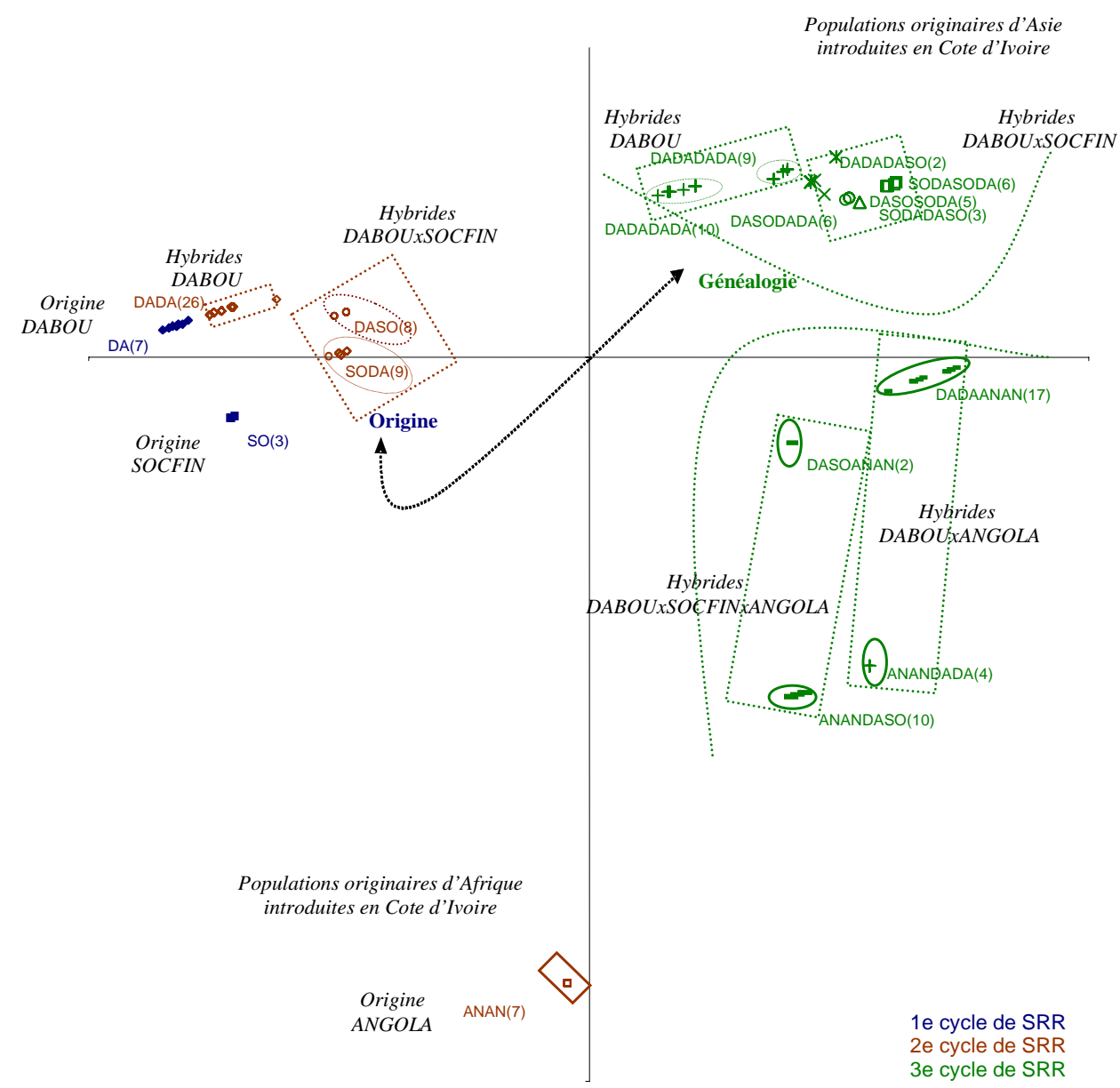

Figure 2: Projection des géniteurs sélectionnés dans le plan 1-2 de l'ACP;

Axe 1(Horizontal): Critère généalogique, Axe 2(Vertical): Critère géographique.

\section{3-3. Pouvoir disjonctif du critère "généalogie parentale"}

Dans les huit populations de géniteurs du troisième cycle de SRR, l'inertie à l'intérieur des catégories disjonctives révélée par la classification automatique a évolué en sept points de disjonction (Figure 3). 


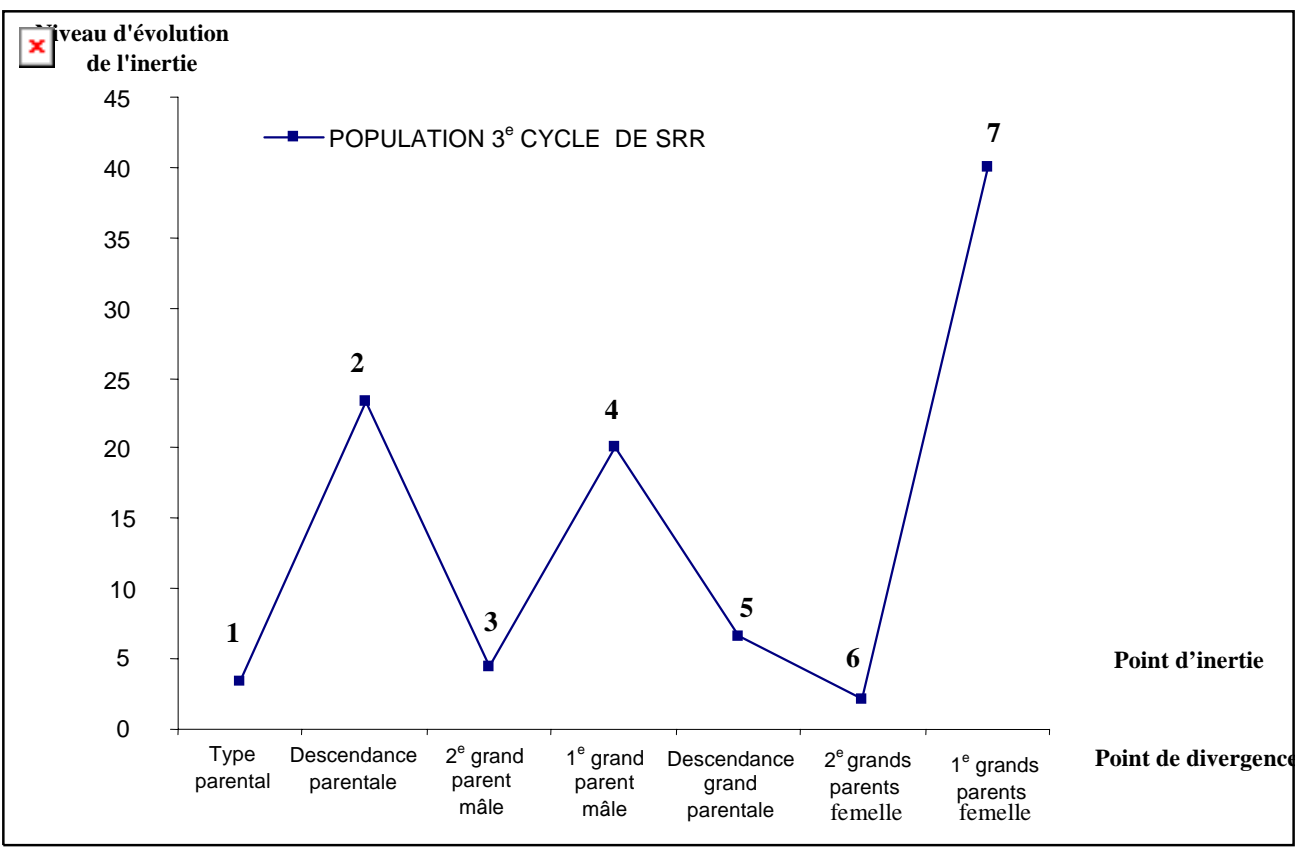

Figure 3 : Evolution des points d'inertie de la classification automatique des populations de géniteurs du troisième cycle de SRR chez le palmier à huile.

Parmi ces points de disjonction, trois niveaux de forte divergence ont été identifiés. Le premier niveau de forte divergence est représenté par la "descendance parentale" (point d'inertie 2). Le deuxième niveau est représenté par le génotype du "premier grands-parents mâle" au point d'inertie 4. Le point d'inertie 7 correspond au troisième niveau de forte divergence et est représenté par le génotype du "premier grands-parents femelle". Des catégories disjonctives se sont formées en chacun de ces trois grands points de disjonction. Au point de disjonction 7, les géniteurs hybrides du troisième cycle de SRR dont les premiers grands-parents femelles sont de génotype AN se sont différenciés des géniteurs dont les premiers grands-parents femelles sont de génotype DA.

L'utilisation de la variable catégorielle "premier grands-parents femelle" comme critère généalogique de différenciation des populations a permis d'établir plus de 60 $\%$ des disjonctions totales exprimées dans la population de géniteurs du troisième cycle de SRR (Figure 4). Au point de disjonction 4, les géniteurs hybrides dont les premiers grands-parents mâles sont de génotype DA se sont différenciés des géniteurs hybrides dont les premiers grands-parents mâles sont de génotype SO. La variable "premier grands-parents mâle" a permis d'établir $51 \%$ des disjonctions totales exprimées dans la population de géniteurs du $3^{\mathrm{e}}$ cycle de SRR. Au point de 
disjonction 2, les géniteurs hybrides du $3^{\mathrm{e}}$ cycle de SRR se sont différenciés en 14 catégories disjonctives en fonction des descendances parentales (Figure 4). L'utilisation de la variable "descendance parentale" a permis d'établir $27 \%$ de la variabilité totale exprimée dans la population de géniteurs du 3e cycle de SRR.

Arbre de 31 Observations.

Méthode de Ward

Pourcentage de divergence

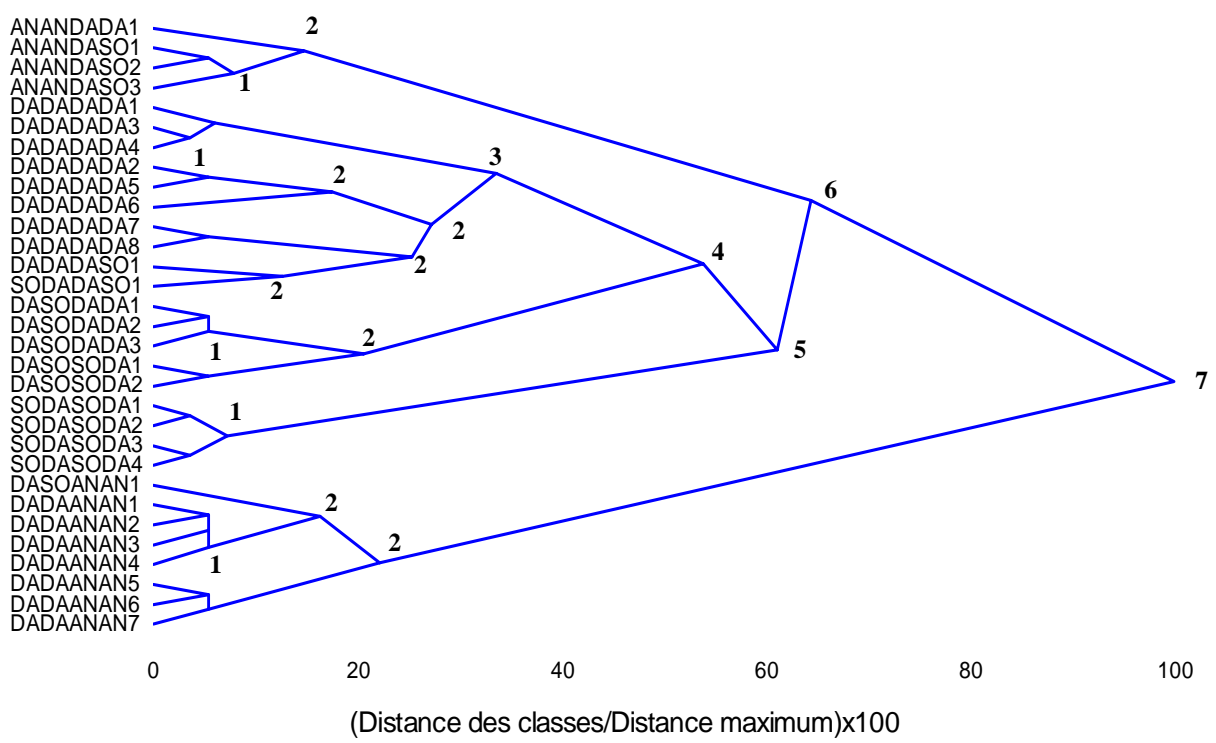

Figure 4 : Classement des géniteurs du $3^{e}$ cycle de SRR en fonction du niveau d'expression de la divergence.

\section{Discussion}

Les pouvoirs disjonctifs des deux critères de disjonction des populations en sélection ont été de $70 \%$ pour le critère "généalogie parentale" et $14 \%$ pour le critère "origine géographique". Ces deux critères ont été des sources de différenciations des populations largement exploitées par les sélectionneurs du palmier à huile. Ces critères sont devenus essentiels pour la sélection des populations de palmier à huile depuis que certaines de ces populations ne peuvent plus être différenciées par les variables agro-morphologiques. En effet, en 1993, JACQUEMARD et al. [16] ont 
indiqué, pour le schéma de SRR, que la disjonction des populations de géniteurs en fonction de leur origine géographique peut permettre d'exploiter une plus grande source de diversité du matériel génétique. Cette observation a aussi été annoncée par MEUNIER et GASCON depuis 1972 [2]. L'utilisation du critère "origine géographique" comme source de diversification du matériel génétique, a souvent été un bon moyen pour évaluer tout nouveau matériel avant son introduction dans le schéma de SRR. En effet, ADON et al. [17] dans le but d'introduire de la diversité génétique dans le matériel en sélection ont utilisé du matériel d'origine ANGOLA comme nouvelle source de diversification des populations de géniteurs sélectionnés. L'exploitation de la disjonction basée sur les origines géographique des populations de palmier à huile a été soutenue par une étude menée par HAYATI et al. en 2004 [8]. Ces derniers ont montrés, à travers une étude de la diversité génétique de 26 populations de palmier à huile issues de prospection dans 10 pays de la zone ouest africaine et de un pays de l'Asie de l'est, l'existence de divergences moléculaires importantes entre populations de différentes origines géographiques. Par conséquent, dans certains programmes d'amélioration génétique en début d'exécution, l'exploitation de l'origine géographique comme critère de disjonction des populations en sélection peut être une alternative à l'utilisation de données moléculaires dont les coûts d'exploitation sont souvent très élevés. Cette étude a aussi montré que le critère "généalogie parentale" peut être utilisé pour exploiter une disjonction plus importante que le critère "origine géographique". En effet, l'exploitation des variables catégorielles élaborées à partir de critère généalogique reste le moyen le plus efficace pour établir des différences entre géniteurs sélectionnés. Pour mieux différencier les populations de géniteurs sélectionnés, le critère "généalogie parentale" peut être associé au critère "origine géographique". Ainsi, DURAND-GASSELIN et al. [10], en plus des origines géographiques des grandsparents ont tenu compte des généalogies parentales afin d'établir des différences dans les populations de géniteurs du deuxième cycle. L'utilisation de ces deux critères de disjonction a permis à ces derniers de différencier rapidement certaines populations qui ont les mêmes performances agronomiques. L'exploitation de certaines variables catégorielles peut donc permettre d'orienter le choix vers certains populations de géniteurs non pas sur la base de leurs performance agronomiques mais sur les connaissances des caractéristiques des grands-parents. Cette méthode de choix de géniteurs peut être proposée comme méthode de sélection en test indirect dans la mesure où les caractéristiques agronomiques et moléculaires des grands-parents sont très bien connues. Les analyses statistiques des variables catégorielles liées à la généalogie parentale sont devenues 
indispensables pour donner à la méthode de sélection de géniteurs par test indirect un fondement non empirique.

\section{Conclusion}

L'utilisation des variables catégorielles comme critère de différenciation du matériel sélectionné est une bonne alternative lorsque certaines données agromorphologiques ne parviennent plus à établir de différences entre populations de géniteurs sélection. Cependant, l'utilisation de ce moyen de différenciation ne peut se faire sans tenir compte du pouvoir de disjonction exprimé par chaque variable catégorielle. Cette étude a montré que l'exploitation d'une source de divergence établie au moyen de critères géographiques est moins expressive que celle liée aux critères généalogiques. Dans les cycles de sélection avancés, la meilleure expression de divergence peut être établie avec la combinaison des critères "origine géographique" et "généalogie parentale" ou exclusivement avec le critère "généalogie parentale". L'adoption de nouveau critère de divergence catégorielle comme alternative à l'utilisation de la divergence moléculaire doit être soumise à une analyse statistique des variables catégorielles et être étendue à l'ensemble des populations de géniteurs sélectionnés de palmier à huile de la Côte d'Ivoire.

\section{Références}

[1] - FA0, Le palmier à huile au kenya., Focus/2002, (2002) http://www.fao.org/ag/fr/magazine/0202spl.h

[2] - J. MEUNIER, J.P. GASCON. Le schéma général d'amélioration du palmier à huile à I'IRHO. Oléagineux, 27 (1) (1972) 1-12.

[3] - B. ADON. Evaluation des introductions de palmier à huile (Elaeis guineensis Jacq.): Utilisation dans le schéma de sélection récurrente réciproque. Thèse de: Université de Cocody, Abidjan, (1995) $117 \mathrm{p}$.

[4] - T.T.V. CA0. Organisation de la variabilité génétique chez le palmier à huile (Elaeis guineensis Jacq.). Conséquences pour l'amélioration des populations et de la création variétale. Thèse de: Instutut National Agronomique, Montpellier, (1995) $200 \mathrm{p}$.

[5] - C. BAKOUME, B. ADON, B. COCHARD, F. POItIER, T. DURAND-GASSELIN, P. AMBLARD. Assessment of yocoboue wild oil palm (Elaeis quineensis Jacq.) from Côte d'Ivoire. Euphtica, 121 (2001) 59-6 
[6] - E. BARCELOS. Etude de la diversité génétique du genre Elaeis (E. oleifera (Kunth) Cortès et E. guineensis Jacq.) par marqueurs moléculaires (RFLP et AFLP) Thèse de : Ecole supérieure agronomique de Montpellier, Montpellier, France (1998) $138 p$

[7] - N. BILLOTTE, A.M. RISTERUCCI, E. BARCELOS, J.P. NOYER, P. AMBLARD, F.C. BAURENS. Development, characterisation, and across-taxa utility of oil palm (Elaeis guineensis Jacq.) Microsatellite markers. Genome 44 (2001) 413-425.

[8] - A. HAYATI, R. WICKNESWARI, I. MAIZURA, N. RAJANAIDU. Genetic diversity of oil palm ( Elaeis guineensis Jacq.) germplasm collections from Africa: implications for improvement and conservation of genetic resources, TAG Theoretical and Applied Genetics, 108,(7) (2004) 1274-1284

[9] - C.W.S. HARTLEY. The oil palm (Elaeis guineensis Jacq.).Ed. 1, (Tropical Agriculture Series, Essex), Londres, Royaume-Uni, LONGMAN (1967) $762 \mathrm{p}$.

[10] - T. D URAND-GASSELIN, L. BAUDOUIN, B. COCHARD, B. ADON, V. CAO THI TUAANG. Stratégies d'amélioration génétique du palmier à huile. Plantation, Recherche, Développement, 6 (5) (1999) 344-355.

[11] - T. DURAND-GASSELIN, R. KOUAME KOUAME, B. COCHARD, B. ADON, P. AMBLARD. Diffusion variétale du palmier à huile (Elaeis guineensis jacq.). OCL, 7 (2) (2000) 207214.

[12] - C. LEROUX-SCRIBE. L'analyse des données: Les guides SAS. EUROPSTAT, (Tomel), France, (1997) $398 \mathrm{p}$.

[13]- J.D. JOBSON. Applied multivariate data analysis. Volume II: categorical and multivariate methods. Springer-Verlag, New York (1992) 345-388, 483-568.

[14] - L. LEBART, A. MORINEAU and M. PIRON. Statistique exploratoire multidimensionnelle. 2ème édition. Dunod, Paris (1997) 155-206.

[15] - TATSOFT Inc. STATISTICA (data analysis software system), version 5.1 (2001). Www.statsoft.com.

[16] - J.C. JACQUEMARD, B. KOUAME, J. MEUNIER. Un exemple de stratégie de sélection récurrente réciproque: le palmier à huile, Elaeis quineensis Jacq. AUPELF-UREF (28) Ed. JOHN LIBBEY EUROTEXT Paris, France, (1993) 371-384.

[17] - B. ADON, L. BAUDOUIN, T. DURAND-GASSELIN, B. KOUAME. Utilisation de matériel non amélioré pour la sélection du palmier à huile: l'origine Angola. Plantation, Recherche, Développement, Sélection (mai-juin) (1998) 201-205. 